\title{
黄帚䝴吾花序结构的资源配置与环境的关系
}

\author{
刘左军 ${ }^{1.2}$ 杜国祯 ${ }^{*}$ 陈家宽 ${ }^{3}$ \\ (1 兰州大学干旱农业生态国家重点实验室, 兰州 730000) \\ (2 西北师范大学生命科学学院, 兰州 730070) \\ (3 复旦大学生物多样性与生态工程教育部重点实验室, 上海 200433)
}

\begin{abstract}
摘 要 为了系统地了解黄帚橐吾 (Ligularia virgaurea) 自然种群的繁殖对策, 在其繁殖分配研究的基础上, 通过统 计不同部位头状花序的生物量投入，进一步分析了存在于总状花序内资源分配上的结构效应及其对不同生境条件 的反应。结果表明: : 1)总状花序、不同部位头状花序在资源投入上受其栖息生境各种生态因子的影响而在不同生 境和平均种群密度间存在着差异, 并表现出一定的趋势 ,2) 可代表个体水平的总状花序大小、头状花序大小、头状 花序数量和头状花序平均重量等特征, 都与种群密度呈现出程度不同的负相关关系, 而总状花序内不同部位的头 状花序大小则与种群密度变化无关 3)总状花序内的资源分配存在着位置依赖性, 这种显著差异表现为顶部头状 花序比基部和中部头状花序有较大的资源分配 ; ) 总状花序顶部、中部和基部头状花序的资源分配与总状花序大 小表现出极显著或显著的负相关关系。
\end{abstract}

关键词 黄帚㯻吾 花序 资源分配 位置依赖

\section{RELATIONSHIP BETWEEN HABITATS AND RESOURCE ALLOCATION OF INFLORESCENCE STRUCTURE IN LIGULARIA VIRGAUREA}

\author{
LIU Zuo-Jun ${ }^{1,2}$ DU Guo-Zhen ${ }^{1 *}$ and CHEN Jia-Kuan ${ }^{3}$ \\ (1 State Key Laboratory of Arid Agroecology , Lanzhou University , Lanzhou 730000 , China) \\ (2 College of Life Sciences, Northwest Normal University, Lanzhou 730070 , China) \\ (3 Key Laboratory of MOE for Biodiversity Science \& Ecological Engineering , Fudan University, Shanghai 200443 , China)
}

\begin{abstract}
In this study, we investigated resources allocation in intra-inflorescence of Ligularia virgaurea in different habitats and population densities based on the characters of reproductive allocation that have been studied. The material we studied was collected from Nima $\left(101^{\circ} 53^{\prime} \mathrm{E}, 35^{\circ} 58^{\prime} \mathrm{N}\right.$, altitude $\left.3500 \mathrm{~m}\right)$ in Maqu county , Gansu province , in the first turn of Yellow River, North-East region of Tibetan Plateau. This region belongs to the alpine meadow rangeland type. The plot contained habitats of different degrees of degradation. $L$. virgaurea is a perennial herb of Compositae. The flowering phase lasts from July to August, with blooming starting at the top of the raceme. The frutescence is in September, about 10 days. It is the dominant weed in alpine meadow now because of the toxic milky material, selective predation and over-grazing by livestock. The aim of our research is to reveal the reproductive strategies of $L$. virgaurea at the level of the inflorescence.
\end{abstract}

During the latter period of seed maturation of L. virgaurea in September 2000 , we sampled randomly from three habitats, floodland, hillside and lowland, and two populations of different density in each habitat. There were 10-16 quadrates per plot, 1-3 seed producing ramets per quadrate were sampled, and brought to the laboratory. The vegetative and reproductive structures were separated, heated 24 hours in $75{ }^{\circ} \mathrm{C}$ drying oven , and weighed using an electronic balance $(\mathrm{g} / 10000)$. The ramet , raceme , capitula of different positions on the raceme, and the number of capitula per raceme were measured. The data were analyzed by ANOVA and linear regression. The measures are : the size of ramet and raceme represented by their biomass ; mean weights of capitula (the ratio of biomass and numbers of capitula per raceme) ; the available capitula of top , middle and bottom represents separately the top , the position of $1 / 2 \mathrm{RI}$ and the bottom ; the resource allocation of capitula at different position means the biomass percent of capitula / raceme ; available capitulum means it could produce seeds, unavailable capitulum means it did not.

The results show : 1) different habitats or population densities differ in resource investment of raceme and capitula of different positions within raceme, and it exhibits some trends as follows : floodland > hillside > lowland, uncovered land > covered land, and low population density > high population density ;2) although most of the correlation coefficients are small, there are negative correlations between the characteristics of 
racemes ( such as the size of raceme and capitula , the number and mean weight of capitulum) and population densities, and no correlations between the size of capitula of different positions within the raceme and population densities ; 3 ) there is a position-based effect within inflorescences, such as the significant decrease in the resource investment between the early-formed or top capitulum of the raceme and later-formed bottom or middle capitula of the raceme ;4) there are significant negative correlations between the size of the raceme and the allocation of capitula at different positions of the raceme.

Key words Ligularia virgaurea , Inflorescence, Resource allocation , Position-based

植物的繁殖分配是其生活史至关重要的特征, 而在诸多的影响因子中，繁殖体大小与个体大小的 关系被认为是最基本的研究内容 (Weiner, 1988; Klinkhamer et al ., 1992 ;Schmid \& Weiner, 1993)。花 序作为植物有性繁殖器官, 其大小与结构对植物的 繁殖效率、交配系统的进化有着十分重要的意义，倍 受植物学家、生态学家和进化生物学家的关注, 而有 关花序的大量研究报道也充分说明了这一点 (Willson \& Price , 1977 ;Cunningham , 1997 ;Fishbein \& Venable , 1996 ;Brody \& Mitchell , 1997 ;Bradley et al. , 1997 ;Tucker \& Grimes , 1999 ;Singer et al . , 1999 ;Bayer , 1999 ;Emms et al., 1997 ; 张大勇等，2001)。

由不同数量的花 (雌花、雄花和两性花) 或亚单 位 (如小穗)组成的花序在各组成成分 (花、小穗、种 子)之间同样存在着不同程度的资源分配和竞争问 题 造成花序不同部位间的组成成分在数量、大小、 发育、形态乃至繁殖效率上的差异，从而在不同层次 水平上反映出了植物在不同生境条件下的繁殖对策 (Cunningham , 1997 ;Warringa et al ., 1998 ;Ashman \& Hitchens , 2000 ;Ashman et al ，2001)。此外，花序内 的这种资源分配模式既受到其内在遗传基因的直接 调控 (Komatsu et al ., 2001 ; Singer et al ., 1999 ;Maes et al . , 1999 ;Souer et al ， 1998) ,也受到生态因子的 间接影响(Cunningham，1997），并与个体对其它生理 功能 (储存、无性增殖、未来生殖) 的资源分配相关联 (Cunningham , 1997 ;Proctor et al. , 1999 ;Germán et al . ,2001)。

我们选择高寒草甸退化草地标志性毒草一一黄 帚革吾 (Ligularia virgaurea) 的总状花序为实验材料, 在了解其自然种群繁殖分配特征的基础上 (刘左军 等 2002），进一步从个体和种群两个水平上探讨:1) 此类复合花序内亚单位间的资源分配特征及其位置 效应 2)不同生境条件是否对这种资源分配特征及 其位置效应产生影响；3)这种资源分配特征及其位 置效应与无性系株大小的关系;4) 此类复合花序内 的资源分配与其它类型的有何差异。其目的是在进 行理论问题研究的同时, 进一步了解和掌握黄帚革
吾繁殖对策, 为寻求有效的的防治途径提供理论依 据。

\section{1 材料和方法}

\section{1 研究地点与自然概况}

甘肃省甘南藏族自治区玛曲县 (东经 $101^{\circ} 53^{\prime}$, 北纬 $35^{\circ} 58^{\prime}$, 平均海拔 $3500 \mathrm{~m}$ ) 位于青藏高原东北 缘, 甘肃省最南端, 黄河第一弯。其东南部与四川 省,西南部和西部与青海省接壤。该区地势高六, 地 表开阔,多风, 年平均气温 $1.2{ }^{\circ} \mathrm{C}$, 年降水量 620 $\mathrm{mm}$ 。由于该区植被茂密, 群落结构复杂, 可食牧草 产量高, 是优良的天然牧场。属高寒草甸植被类型 (吴征镒,1980)。但近 20 多年来,由于人口和牲畜 数量的持续增长, 而草场利用观念落后 草场科学化 管理未能及时跟上, 使草场过牧现象日趋加重。结 果导致草场群落结构发生了深刻的变化, 其显著特 征是毒杂草成分显著增加。如黄帚革吾盖度由 20 世纪 70 年代末的 5\% 20\% 上升到目前的 $20 \%$ $50 \%$ 。本研究于 2000 年 8 月 31 日至 9 月 6 日在甘 肃省玛曲县尼玛乡天然草地进行。样地为退化程度 不同的草地。

\section{2 实验材料}

黄帚革吾属菊科革吾属一种多年生草本植物。 因在进行有性繁殖前有较长的营养生长期, 故需要 $4 \sim 6$ 年完成生活史。营养生长期无茎, 仅有 $1 \sim 10$ 片簇生叶, 仅在有性繁殖期有茎。总状花序顶生, 由 几个至数十个头状花序沿总状花序轴辐射状排列组 成, 平均长度为 $10.4 \sim 21.0 \mathrm{~cm}$ (刘左军等, 2002)。 连续两年的野外定株观察, 发现黄帚䝴吾自然种群 每年花期从 7 月上旬见花至 8 月中旬花期结束, 约 $50 \mathrm{~d}$ 左右。一个头状花序的花期约 $10 \mathrm{~d}$ 左右, 总状 花序的开花次序是由顶部头状花序起始到基部头状 花序结束, 花期约 $25 \mathrm{~d}$ 左右。花序成熟期为 9 月中 下旬。除有性繁殖外, 还可通过地下横走的根状茎 进行克隆生长(单保庆等,2000)。由于其含有白色 有毒乳液, 加之过牧和牲畜的选择性取食, 现已成为 高寒草甸一种优势毒杂草。 


\section{3 实验方法}

在黄帚革吾种子成熟后期, 从滩地、沙地和坡地 3 种生境及每一生境黄帚革吾两种不同平均密度的 自然种群中随机取样, 样地概况、取样数及平均密度 详见另文(刘左军等, 2002)。每一样地有 $10 \sim 16$ 个 样方, 每一样方各取 1 3 株结实的无性系株地上部 分, 并将营养结构和生殖结构分别装袋晾干, 带回实 验室。在称干重之前, 先在 $75{ }^{\circ} \mathrm{C}$ 烘箱中烘 $24 \mathrm{~h}$, 然 后再用万分之一天平称重。分别记录无性系株、总 状花序、头状花序以及总状花序顶部、中部、基部等 不同部位头状花序的生物量, 统计单株头状花序数 量。数据通过方差分析和线性回归分析 (杜荣寒, 1999)。测定标准 :黄帚橐吾地上无性系株大小以地 上生物量干重为准;总状花序大小是指基部第一个 有效头状花序以上总状花序的干重; 头状花序均重 为单株头状花序干重与其头状花序数量之比;顶部、 中部、基部头状花序分别指总状花序顶端第一个、总
状花序 $1 / 2$ 处和基端最后一个有效头状花序; 顶部 (或中部、基部) 头状花序资源分配是指顶部 (或中 部、基部) 1 个头状花序生物量占总状花序生物量的 百分数; 有效头状花序指可开花结籽的头状花序, 无 效头状花序则指未开花的头状花序。

\section{2 结果与分析}

2.1 不同生境和种群密度对总状花序以及不同部 位头状花序资源投入的影响

不同生境与密度下, 黄帚革吾总状花序大小、单 株头状花序大小、单株头状花序数量、单株头状花序 均重、总状花序上同一部位 (顶部、中部、基部)头状 花序大小等特征经方差分析，样地间存在着极显著 的差异。再经 Duncan 多重检验，各样地间差异程度 详见表 1 和表 2 。从表 1 和表 2 中可知 :上述特征的 变化共同表现出与生境有关 (依平均大小或数量排 序 :沙地 > 坡地 > 滩地) ;在同一生境内，除沙地外，

表 1 不同生境或密度对黄帚㯻吾总状花序资源分配的影响

Table 1 Effects of different habitats and density on resource allocation of raceme in Ligularia virgaurea

\begin{tabular}{|c|c|c|c|c|c|c|c|c|c|c|c|c|c|}
\hline \multirow{3}{*}{$\begin{array}{c}\text { 样地 } \\
\text { Plot } \\
\text { A }\end{array}$} & \multirow{3}{*}{$\begin{array}{c}\begin{array}{c}\text { 取样数 } \\
\text { Sample } \\
\text { number }\end{array} \\
25\end{array}$} & \multicolumn{3}{|c|}{$\begin{array}{c}\text { 总状花序大小 } \\
\text { Raceme size } \\
\left(\mathrm{g} \text { ind } .^{-1}\right) \\
\end{array}$} & \multicolumn{3}{|c|}{$\begin{array}{c}\text { 头状花序总重 } \\
\text { Capitulum gross weight } \\
\left(\mathrm{g} \text { ind. }{ }^{-1}\right)\end{array}$} & \multicolumn{3}{|c|}{$\begin{array}{c}\text { 头状花序数量 } \\
\text { Capitulum number }\end{array}$} & \multicolumn{3}{|c|}{$\begin{array}{c}\text { 头状花序均重 } \\
\text { Capitulum mean weight } \\
\left(\mathrm{g} 100 \text { ind. }^{-1}\right)\end{array}$} \\
\hline & & \multirow{2}{*}{$\frac{\mathrm{M} \pm \mathrm{SD}}{3.17 \pm 1.54}$} & \multicolumn{2}{|c|}{ MC } & \multirow{2}{*}{$\frac{\mathrm{M} \pm \mathrm{SD}}{2.27 \pm 0.94}$} & \multicolumn{2}{|c|}{ MC } & \multirow{2}{*}{$\frac{M \pm S D}{31.84 \pm 10.00}$} & \multicolumn{2}{|c|}{$\mathrm{MC}$} & \multirow{2}{*}{$\frac{\mathrm{M} \pm \mathrm{SD}}{7.16 \pm 1.65}$} & \multicolumn{2}{|c|}{ MC } \\
\hline & & & A & a & & A & $\mathrm{a}$ & & A & a & & A & $\mathrm{a}$ \\
\hline B & 25 & $1.24 \pm 0.57$ & $\mathrm{DE}$ & def & $0.97 \pm 0.43$ & $\mathrm{CDE}$ & de & $21.16 \pm 6.48$ & $\mathrm{CDEF}$ & def & $4.51 \pm 1.26$ & CD & cde \\
\hline C & 25 & $1.77 \pm 0.93$ & $\mathrm{CD}$ & $\mathrm{cd}$ & $1.34 \pm 0.68$ & $\mathrm{CD}$ & $\mathrm{cd}$ & $27.56 \pm 10.47$ & $\mathrm{ABC}$ & $a b c$ & $4.91 \pm 1.75$ & $\mathrm{BCD}$ & $\mathrm{cd}$ \\
\hline D & 25 & $2.03 \pm 1.13$ & $\mathrm{BC}$ & $\mathrm{cd}$ & $1.45 \pm 0.70$ & C & c & $24.52 \pm 8.85$ & BCD & bed & $5.82 \pm 1.21$ & B & $\mathrm{b}$ \\
\hline $\mathrm{E}$ & 25 & $0.88 \pm 0.35$ & $\mathrm{E}$ & $\mathrm{f}$ & $0.69 \pm 0.27$ & $\mathrm{E}$ & $\mathrm{f}$ & $17.88 \pm 5.12$ & $\mathrm{~F}$ & $\mathrm{f}$ & $3.78 \pm 0.86$ & $\mathrm{D}$ & e \\
\hline $\mathrm{F}$ & 25 & $1.33 \pm 0.52$ & $\mathrm{DE}$ & def & $1.06 \pm 0.41$ & $\mathrm{CDE}$ & de & $24.52 \pm 8.42$ & BCDE & bcde & $4.43 \pm 1.32$ & CD & cde \\
\hline G & 25 & $1.48 \pm 0.61$ & $\mathrm{CDE}$ & cde & $1.11 \pm 0.41$ & $\mathrm{CDE}$ & cde & $22.04 \pm 5.07$ & CDEF & def & $4.95 \pm 1.07$ & BC & c \\
\hline $\mathrm{H}$ & 25 & $2.69 \pm 1.32$ & $\mathrm{AB}$ & $\mathrm{ab}$ & $2.03 \pm 0.94$ & $\mathrm{AB}$ & $\mathrm{ab}$ & $28.80 \pm 9.80$ & $\mathrm{AB}$ & $\mathrm{ab}$ & $7.08 \pm 2.20$ & A & $\mathrm{a}$ \\
\hline
\end{tabular}

$\mathrm{M}$ 平均数 Mean $\mathrm{SD}$ 标准差 Standard deviation $\mathrm{MC}$ :多重比较 Multiple comparison 同一列字母相同者, 为差异不显著; 字母不同者, 大写字 母表示差异极显著 $(p<0.01)$ ，小写字母表示差异显著 $(p<0.05)$ Data with the same letter are not significant difference $(p>0.05)$, ones with different letters are significant difference, small letter means significant level $(p<0.05)$ and capital letter means high significant level $(p<0.01)$ in each column

表 2 不同生境或密度对总状花序同一部位头状花序大小的影响

Table 2 Effects of different habitat and density on capitulum size of the same position of raceme in Ligularia virgaurea

\begin{tabular}{|c|c|c|c|c|c|c|c|c|c|c|}
\hline \multirow{3}{*}{$\begin{array}{c}\text { 样地 } \\
\text { Plot }\end{array}$} & \multirow{3}{*}{ 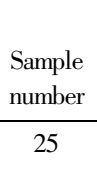 } & \multicolumn{3}{|c|}{$\begin{array}{c}\text { 顶部头状花序 } \\
\text { Top capitulum (g) }\end{array}$} & \multicolumn{3}{|c|}{$\begin{array}{c}\text { 中部头状花序 } \\
\text { Middle capitulum (g) }\end{array}$} & \multicolumn{3}{|c|}{$\begin{array}{c}\text { 基部头状花序 } \\
\text { Bottom capitulum (g) }\end{array}$} \\
\hline & & \multirow{2}{*}{$\frac{\mathrm{M} \pm \mathrm{SD}}{9.09 \pm 2.49}$} & \multicolumn{2}{|c|}{$\mathrm{MC}$} & \multirow{2}{*}{$\begin{array}{c}\mathrm{M} \pm \mathrm{SD} \\
7.08 \pm 2.01\end{array}$} & \multicolumn{2}{|c|}{$\mathrm{MC}$} & \multirow{2}{*}{$\begin{array}{c}\mathrm{M} \pm \mathrm{SD} \\
5.45 \pm 2.19\end{array}$} & \multicolumn{2}{|c|}{$\mathrm{MC}$} \\
\hline & & & $\mathrm{AB}$ & $a b$ & & $\mathrm{AB}$ & $a b$ & & $\mathrm{AB}$ & $a b$ \\
\hline B & 25 & $5.95 \pm 2.08$ & $\mathrm{CD}$ & de & $5.02 \pm 1.42$ & $\mathrm{DE}$ & de & $4.02 \pm 1.58$ & $\mathrm{CD}$ & $\mathrm{cd}$ \\
\hline C & 25 & $5.94 \pm 2.57$ & $\mathrm{CD}$ & de & $5.38 \pm 1.99$ & CD & d & $4.07 \pm 1.88$ & $\mathrm{CD}$ & $\mathrm{cd}$ \\
\hline $\mathrm{D}$ & 25 & $7.67 \pm 3.42$ & $\mathrm{ABC}$ & bc & $6.47 \pm 1.81$ & $\mathrm{ABC}$ & bc & $5.00 \pm 2.50$ & $\mathrm{ABC}$ & bc \\
\hline $\mathrm{E}$ & 25 & $5.20 \pm 2.07$ & $\mathrm{D}$ & $\mathrm{e}$ & $3.85 \pm 1.13$ & $\mathrm{E}$ & $\mathrm{f}$ & $3.09 \pm 0.97$ & $\mathrm{D}$ & d \\
\hline $\mathrm{F}$ & 25 & $5.98 \pm 1.77$ & $\mathrm{CD}$ & de & $4.67 \pm 1.64$ & $\mathrm{DE}$ & de & $3.85 \pm 1.02$ & $\mathrm{D}$ & d \\
\hline G & 25 & $7.04 \pm 1.88$ & $\mathrm{CD}$ & $\mathrm{cd}$ & $4.64 \pm 1.01$ & $\mathrm{DE}$ & de & $4.05 \pm 1.16$ & $\mathrm{CD}$ & $\mathrm{cd}$ \\
\hline $\mathrm{H}$ & 25 & $9.56 \pm 3.71$ & A & $\mathrm{a}$ & $7.72 \pm 2.27$ & $\mathrm{~A}$ & $\mathrm{a}$ & $6.02 \pm 1.90$ & A & $\mathrm{a}$ \\
\hline
\end{tabular}

M , SD , MC :同表 1 See Table 1 
与生境内该种群平均密度有关 (低密度 $>$ 高密度), 并在低密度下也与样地植被状况有关 (裸地 $>$ 非裸 地)。而在沙地生境内，上述特征平均值表现出高密 度种群 > 低密度种群。产生这一现象的原因是沙地 植被盖度低, 可食牧草少, 尤其是黄帚革吾种群密度 较高时, 甚至无牧草可食, 而黄帚䝴吾又为毒草。因 此, 家畜对黄帚革吾高密度分布区域的干扰程度低 于低密度分布区域，导致高密度分布区域的个体由 于受损很少或无损伤, 则相对有较多的资源分配给 总状花序上, 从而表现出上述现象。由此可见, 黄帚 革吾总状花序内资源分配模式受栖息生境条件、自 然种群平均密度以及牲畜选择性受食行为的影响。 由于上述特征具有无性系株大小的依赖性, 因而其
差异是不同生境和种群密度对资源投入间接影响的 结果。

2.2 不同种群密度与总状花序及不同部位头状花 序资源投入的关系

同一生境条件下,黄帚㯻吾总状花序大小、单株 头状花序大小、单株头状花序数量、单株头状花序均 重等特征均与该生境内种群密度的变化表现出程度 不同的负相关关系，仅在少数样地内达到显著或极 显著水平(图 1 ,图 2)。而总状花序上同一部位(顶 部、中部、基部) 头状花序大小则与种群密度无关。 这说明密度对黄帚革吾总状花序资源投入的影响也 可发生在个体水平上，而在总状花序内部与某一部 位头状花序的投入无关，即无密度依赖性。
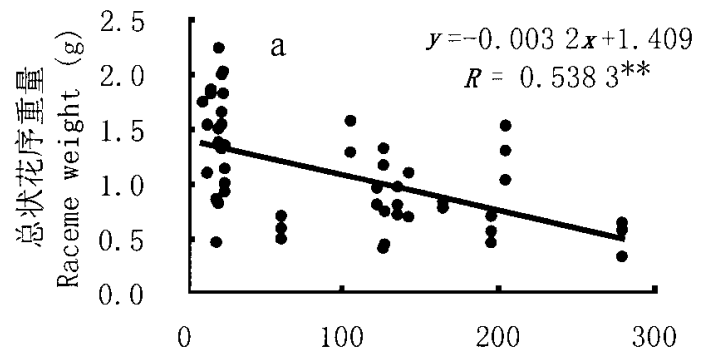

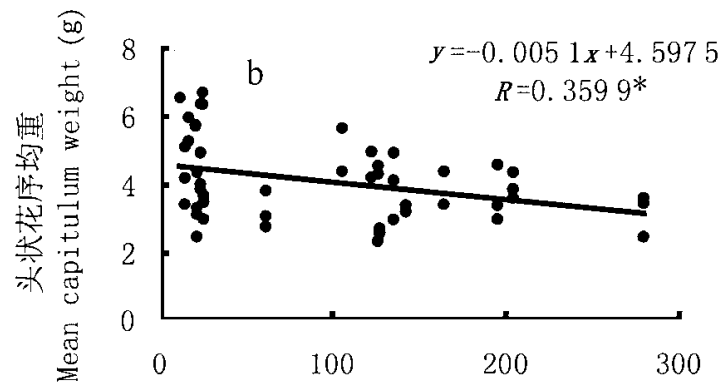

密度 Density (ind. $\cdot \mathrm{m}^{-2}$ )

图 1 样地 $(E+F)$ : 密度与总状花序大小 (a)及头状花序均重 $(b)$ 的关系

Fig. 1 Plot $(\mathrm{E}+\mathrm{F})$ : The relationship of density and raceme (a) or capitulum mean weight $(\mathrm{b})$
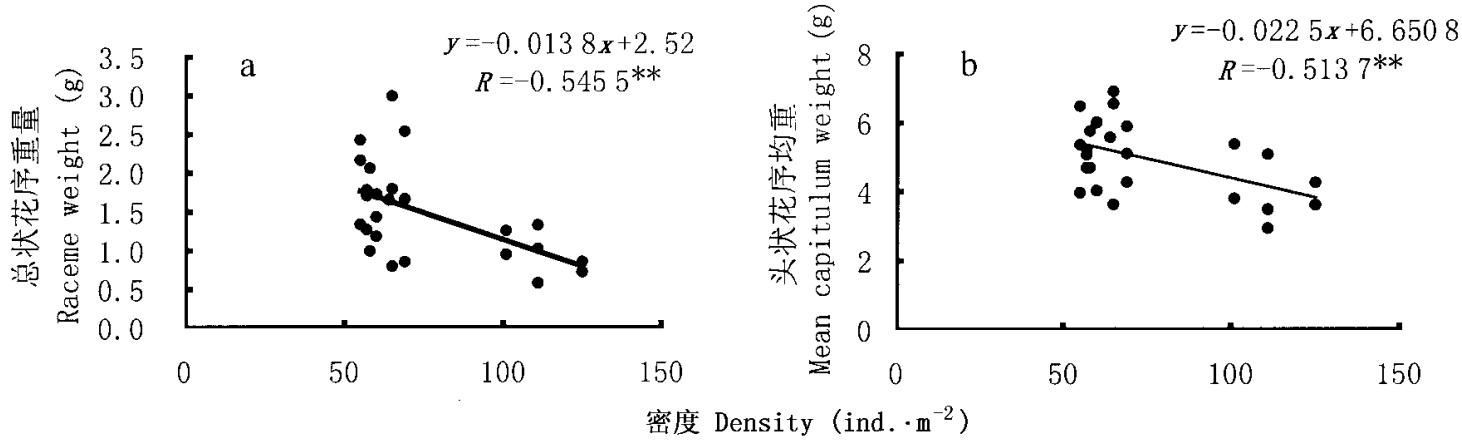

图 2 样地 $\mathrm{G}$ : 密度与总状花序大小 (a)及头状花序均重 $(\mathrm{b})$ 的关系

Fig.2 Plot G : The relationship of density and raceme (a) or capitulum mean weight (b)

\section{3 不同部位头状花序间的资源分配}

在不同生境和种群密度中, 同一总状花序上不 同部位头状花序间的资源分配共同表现出顶部 > 中 部 > 基部的趋势。这种差异经方差分析, 除样地 $\mathrm{F}$ 外 样地 C、D 达到显著或样地 $A 、 B 、 E 、 G 、 H$ 达到极 显著的水平 (表 3 )。由此可见, 不同的生境和密度 对这种同一总状花序上不同部位间头状花序资源分 配的影响有所不同(沙地、滩地 > 坡地)。就顶部、中
部、基部头状花序间这种资源分配差异而言，主要体 现在顶部与基部头状花序之间，如样地 $A 、 B 、 D 、 E 、$ $\mathrm{G} 、 \mathrm{H}$ 中差异极显著，样地 $\mathrm{C}$ 中为显著。其次是顶部 与中部头状花序之间, 如样地 $A 、 E 、 G$ 中差异极显 著 样地 $\mathrm{H}$ 中差异为显著。最后是中部与基部头状 花序之间，如样地 $\mathrm{A} 、 \mathrm{H}$ 中差异为显著 (表 3)。

2.4 总状花序资源投入与无性系株大小的关系 不同的生境和种群密度下, 黄帚䝴吾总状花序 
大小、单株头状花序大小 (即繁殖生物量) (刘左军 等, 2002)、单株头状花序数量以及单株头状花序均 重等特征均与无性系株大小呈正相关关系，绝大多 数达到极显著水平, 少数为显著水平 (表 4 )。由此 可见 随着无性系株大小的增加, 由于头状花序数和 头状花序均重都在增加，从而使总状花序大小与无
性系株大小的相关程度最高。

\section{5 不同部位头状花序资源分配与总状花序大小 的关系}

在不同生境和密度中, 不同部位头状花序的资 源分配与总状花序大小均表现出负相关关系，但相 关程度有所不同 (表5)。如中部、顶部头状花序的

表 3 不同部位头状花序资源分配的比较

Table 3 Comparison of resource allocation of capitula of different positions

\begin{tabular}{|c|c|c|c|c|c|c|c|c|c|c|c|}
\hline $\begin{array}{c}\text { 样地 } \\
\text { Plot }\end{array}$ & $\begin{array}{c}\text { 部位 } \\
\text { Position }\end{array}$ & $\begin{array}{c}\text { 资源分配 } \\
\text { RA }(\%)\end{array}$ & SD & & & $\begin{array}{c}\text { 样地 } \\
\text { Plot }\end{array}$ & $\begin{array}{c}\text { 部位 } \\
\text { Position }\end{array}$ & $\begin{array}{c}\text { 资源分配 } \\
\operatorname{RA}(\%)\end{array}$ & SD & & \\
\hline \multirow{3}{*}{ A } & 顶部 Top & 4.3850 & \pm 1.52 & A & a & \multirow{3}{*}{$\mathrm{E}$} & 顶部 Top & 8.1904 & \pm 3.59 & A & $\mathrm{a}$ \\
\hline & 中部 Middle & 3.3212 & \pm 1.02 & B & $\mathrm{b}$ & & 中部 Middle & 6.0277 & \pm 1.79 & B & $\mathrm{b}$ \\
\hline & 基部 Bottom & 2.5387 & \pm 1.04 & B & c & & 基部 Bottom & 4.8794 & \pm 1.52 & B & $\mathrm{b}$ \\
\hline \multirow{3}{*}{ B } & 顶部 Top & 6.9788 & \pm 3.43 & A & a & \multirow{3}{*}{$\mathrm{F}$} & 顶部 Top & 6.6527 & \pm 3.96 & A & a \\
\hline & 中部 Middle & 5.6140 & \pm 2.15 & $\mathrm{AB}$ & $a b$ & & 中部 Middle & 4.9637 & \pm 2.41 & A & a \\
\hline & 基部 Bottom & 4.6360 & \pm 2.11 & B & $\mathrm{b}$ & & 基部 Bottom & 5.0043 & \pm 3.78 & A & a \\
\hline \multirow{3}{*}{$\mathrm{C}$} & 顶部 Top & 5.4014 & \pm 3.23 & A & a & \multirow{3}{*}{ G } & 顶部 Top & 6.8344 & \pm 2.09 & A & a \\
\hline & 中部 Middle & 4.4811 & \pm 1.92 & A & $a b$ & & 中部 Middle & 4.5340 & \pm 1.023 & B & $\mathrm{b}$ \\
\hline & 基部 Bottom & 3.5772 & \pm 2.24 & A & $\mathrm{b}$ & & 基部 Bottom & 3.8848 & \pm 1.15 & B & $\mathrm{b}$ \\
\hline \multirow{3}{*}{$\mathrm{D}$} & 顶部 Top & 6.4778 & \pm 4.18 & A & a & \multirow{3}{*}{$\mathrm{H}$} & 顶部 Top & 5.0924 & \pm 1.74 & A & a \\
\hline & 中部 Middle & 5.2747 & \pm 2.76 & $\mathrm{AB}$ & $a b$ & & 中部 Middle & 4.2447 & \pm 1.44 & $\mathrm{AB}$ & $\mathrm{b}$ \\
\hline & 基部 Bottom & 3.9264 & \pm 1.86 & B & $\mathrm{b}$ & & 基部 Bottom & 3.2827 & \pm 1.21 & B & $\mathrm{c}$ \\
\hline
\end{tabular}

RA 资源分配 Resource allocation SD、MC :同表 1 See Table1 同一列字母相同者, 为差异不显著字母不同者, 大写字母示差异极显著 $(p<0$. $01)$,小写字母示差异显著 $(p<0.05)$ Data with the same letter are not significant difference $(p>0.05)$, ones with different letter are significant difference, small letter means significant level $(p<0.05)$ and capital letters means high significant level $(p<0.01)$ in each column

表 4 黄帚㯻吾无性系株大小 $(x)$ 与总状花序资源投入 $(y)$ 的回归分析

Table 4 The regression analyses between ramet size $(x)$ and resource investment $(y)$ of raceme

\begin{tabular}{|c|c|c|c|c|c|c|}
\hline \multirow{3}{*}{$\begin{array}{c}\text { 样地 } \\
\text { Plot } \\
\text { A }\end{array}$} & \multirow{2}{*}{\multicolumn{2}{|c|}{$\begin{array}{c}\text { 总状花序重 } \\
\text { Raceme weight }(\mathrm{g})\end{array}$}} & \multirow{2}{*}{\multicolumn{2}{|c|}{$\begin{array}{l}\text { 单株头状花序数 } \\
\text { Capitulum number }\end{array}$}} & \multirow{2}{*}{\multicolumn{2}{|c|}{ 单株头状花序均重 }} \\
\hline & & & & & & \\
\hline & $y=0.3519 x+0.2542$ & $R=0.8525^{* *}$ & $y=1.6490 x+18.198$ & $R=0.6143^{* *}$ & $y=0.2392 x+5.1818$ & $R=0.5400^{* *}$ \\
\hline B & $y=0.3967 x-0.2937$ & $R=0.9109^{* *}$ & $y=3.5683 x+7.3045$ & $R=0.7175^{* *}$ & $y=0.6130 x+2.1339$ & $R=0.6322^{* *}$ \\
\hline $\mathrm{C}$ & $y=0.3399 x+0.2699$ & $R=0.9612^{* *}$ & $y=2.0195 x+18.65$ & $R=0.5054^{* *}$ & $y=0.5014 x+2.7019$ & $R=0.7495^{* *}$ \\
\hline $\mathrm{D}$ & $y=0.3306 x+0.0851$ & $R=0.9007^{* *}$ & $y=2.0950 x+12.135$ & $R=0.7286^{* *}$ & $y=0.1923 x+4.6893$ & $R=0.4885^{*}$ \\
\hline $\mathrm{E}$ & $y=0.3511 x+0.0162$ & $R=0.9291^{* *}$ & $y=3.8800 x+8.3905$ & $R=0.6929^{* *}$ & $y=0.6601 x+2.1663$ & $R=0.6990^{* *}$ \\
\hline $\mathrm{F}$ & $y=0.3390 x+0.1057$ & $R=0.8740^{* *}$ & $y=3.0859 x+13.422$ & $R=0.4785^{*}$ & $y=0.5290 x+2.5296$ & $R=0.5217^{* *}$ \\
\hline G & $y=0.2165 x+0.4101$ & $R=0.8135^{* *}$ & $y=0.8940 x+17.61$ & $R=0.4066^{*}$ & $y=0.3738 x+3.1013$ & $R=0.8081^{* *}$ \\
\hline $\mathrm{H}$ & $y=0.2165 x+0.4101$ & $R=0.8135^{* *}$ & $y=1.6330 x+18.652$ & $R=0.5053^{* *}$ & $y=0.5131 x+3.8945$ & $R=0.7086^{* *}$ \\
\hline
\end{tabular}

$* p<0.05 \quad * * p<0.01$

表 5 黄帚革吾总状花序大小 $(x)$ 与不同部位头状花序资源分配 $(y)$ 的回归分析

Table 5 The regression analyses between resource allocation of capitula of different positions $(y)$ and capitulum size $(x)$

\begin{tabular}{clllllll}
\hline $\begin{array}{c}\text { 样地 } \\
\text { Plot }\end{array}$ & \multicolumn{2}{c}{$\begin{array}{c}\text { 顶部头状花序 }(\%) \\
\text { Top capitulum }\end{array}$} & \multicolumn{2}{c}{$\begin{array}{c}\text { 中部头状花序 }(\%) \\
\text { Middle capitulum }\end{array}$} & \multicolumn{1}{c}{$\begin{array}{c}\text { 基部头状花序 }(\%) \\
\text { Bottom capitulum }\end{array}$} \\
\hline $\mathrm{A}$ & $y=-0.619 x+6.344$ & $R=-0.6230^{* *}$ & $y=-0.3745 x+4.5067$ & $R=-0.5624^{* *}$ & $y=-0.2204 x+3.2362$ & $R=-0.3262$ \\
$\mathrm{~B}$ & $y=-3.651 x+11.531$ & $R=-0.6036^{* *}$ & $y=-2.431 x+8.6359$ & $R=-0.6401^{* *}$ & $y=-1.8446 x+6.9357$ & $R=-0.4950^{*}$ \\
$\mathrm{C}$ & $y=-2.0738 x+9.0713$ & $R=-0.5954^{* *}$ & $y=-0.9424 x+6.1488$ & $R=-0.4545^{*}$ & $y=-1.1309 x+5.5786$ & $R=-0.4675^{*}$ \\
$\mathrm{D}$ & $y=-2.3259 x+11.207$ & $R=-0.6266^{* *}$ & $y=-1.486 x+8.296$ & $R=-0.6053^{* *}$ & $y=-0.676 x+5.3008$ & $R=-0.4080^{*}$ \\
$\mathrm{E}$ & $y=-4.8791 x+12.46$ & $R=-0.4693^{*}$ & $y=-3.1246 x+8.7618$ & $R=-0.6030^{* *}$ & $y=-2.7251 x+7.264$ & $R=-0.6206^{* *}$ \\
$\mathrm{~F}$ & $y=-5.4876 x+13.923$ & $R=-0.7017^{* *}$ & $y=-2.9703 x+8.8987$ & $R=-0.6246^{* *}$ & $y=-3.8849 x+9.5186$ & $R=-0.7138^{* *}$ \\
$\mathrm{G}$ & $y=-2.3333 x+10.295$ & $R=-0.6866^{* *}$ & $y=-1.589 x+6.8905$ & $R=-0.7896^{* *}$ & $y=-1.0422 x+5.4305$ & $R=-0.5584^{* *}$ \\
$\mathrm{H}$ & $y=-0.6497 x+6.8428$ & $R=-0.4946^{*}$ & $y=-0.7277 x+6.2051$ & $R=-0.6697^{* *}$ & $y=-0.5332 x+4.7192$ & $R=-0.58144^{* *}$ \\
\hline
\end{tabular}

\footnotetext{
$* p<0.05 \quad * * p<0.01$
} 
资源分配几乎都与总状花序大小呈现出极显著的负 相关关系; 而基部头状花序的资源分配则与总状花 序大小除样地 $\mathrm{A}$ 不显著外, 其余都达到显著( 样地 $B 、 C$ 和 D) 或极显著水平 (样地 $E 、 F 、 G$ 和 $H$ )。这说 明黄帚䝴吾总状花序顶部、中部和基部头状花序的 资源分配整体上随总状花序的增大而减少, 这种现 象在中部和顶部的头状花序上表现得更为突出。

\section{3 讨 论}

3.1 种群密度对总状花序及不同部位头状花序资 源投入的影响

在代表个体水平的特征上，如总状花序大小、头 状花序大小、头状花序数量和头状花序均重等, 都与 种群密度呈现出程度不同的负相关关系。这个结果 与无性系株大小等特征与种群密度的结果一致 (刘) 左军等, 2002) , 也与前人的结果相一致 (Weiner, 1988 ;杜国祯等, 1998; 1999 )。反映出随种群密度的 增加 种内资源竞争加剧, 个体所获资源减少, 从而 导致总状花序等特征在大小或数量上的下降。但由 于上述特征具有无性系株大小依赖性, 使种群密度 通过无性系株大小间接引起上述特征的变化。但总 状花序内不同部位的头状花序大小则与种群密度变 化无关, 这说明种群密度的变化虽间接影响到总状 花序大小的变化, 可这种变化是建立在头状花序数 量和头状花序均重共同变化的基础之上的, 从而混 淆了某一头状花序大小对种群密度的反应, 其结果 也是模棱两可的。

\section{2 不同部位头状花序资源分配的位置依赖性}

已有的研究结果表明, 花序内不同部位间的资 源分配存在着显著的变化趋势 (Lee, 1988 ; Diggle, 1995)。一般顶向发育 (或较早发育) 的花序, 其基部 较顶部有较大的花 (Ashman \& Hitchens, 2000)、较大 或较多的胚珠 (Ashman \& Hitchens, 2000 ; Mazer \& Dawson，2001)、种子和果实 (Warringa et al.，1998; Lavee et al., 1999 ; Medrano et al. ,2000)和较低的 $\mathrm{p} / \mathrm{o}$ 比 (Mazer \& Dawson，2001)等, 而基向发育(或较 后发育)的花序与之恰恰相反 (Brunet, 1996 ; Medrano et al . , 2000)。并且认为这种花序内位置依赖的变 化是基部和顶部发育的花、种子和果实等对资源竞 争的结果。我们的实验结果也证明了花序内存在着 不同部位间资源分配的显著差异, 但这种差异表现 为顶部头状花序较基部和中部头状花序有较大的资 源分配, 即与 Brunet(1996) 和 Medrano 等 (2000) 的结 果一致。目前解释花序内这种位置依赖的资源投入
差异的原因有以下几种假说 : 19) 资源竞争假说”,即 早期形成的或距离营养源较近的果实比后期形成的 或距离营养源更远端的果实占有更多的母体，可利 用资源有限 (Bawa \& Webb，1984;Nakamura，1986； Lee , 1988 ;Guitián , 1994 ;Medrano et al ., 2000) ;2) “非均匀传粉假说”, 即整个花序开花期花粉数量或 质量的欠缺 (Thomson, 1985 ; Lee，1988; Brunet \& Charlesworth，1995）3")结构影响假说”, 即花序内在 的对不同部位花繁殖成功的局限性 (Lee，1988； Wolfe , 1992 ;Brunet , 1996 ;Diggle , 1995；1997)。经 过两年的野外实验和观察, 黄帚革吾虽为完全异花 授粉物种，但根据传粉昆虫种类和访花行为，传粉限 制的可能性较小 (另文发表)，而最有可能的是资源 竞争或结构限制。就资源竞争而言, 顶向发育的花 序,由于基部较顶部距离母体资源库近, 往往可获得 较为充分的可利用资源。但基向发育的花序, 如黄 帚䝴吾总状花序，仅靠距离母体资源库的远近已无 法解释花序内部位间的差异。因此，我们认为花序 内这种位置依赖的资源分配应首先考虑花序的发育 特性, 即在花序发育的起始部位可能存在着某种生 理学的调控机制。哪里生长发育越旺盛, 哪里的资 源需求信号就越强，母体可利用资源的投入也就越 多，这也可能就是资源限制造成花序内位置依赖现 象的直接原因。当然, 这种生理学机制以及黄帚䝴 吾总状花序是否还存在结构影响将有待进一步证 明。

3.3 总状花序大小对不同部位头状花序资源分配 的影响

在不同生境和密度中, 黄帚䝴吾总状花序顶部、 中部和基部头状花序的资源分配均随总状花序的增 大而减少 表现出程度不同的负相关关系。这说明 总状花序增大时,面临着头状花序数量增多与头状 花序均重增加两者之间的权衡 (Trade-offs)。我们的 实验结果是总状花序越大, 具有大小依赖的头状花 序均重和头状花序数量也就越大和越多, 但头状花 序均重的增幅落后于头状花序数量的增幅, 这在中 部和顶部的头状花序上表现得更为突出。可见，尽 管头状花序均重对无性系株大小的依赖性强于头状 花序数量的依赖性, 但个体还是分配更多的资源用 于头状花序数量的增多, 其次才是头状花序均重的 增加。

由于已有的研究已经证明，花序越大，对传粉者 的吸引也就越大 (Ohara \& Higashi, 1994 ;Fishbein \& Venable, 1996 ;Brody \& Mitchell , 1997 ;Anne et al. , 
2000 ;Laura et al . ,2002)。但与此同时, 同株异花受 粉也就越多 (Harder \& Barrett, 1995 ;Laura et al. , 2002)。这对完全异花受粉的黄帚革吾来说将产生 什么影响还有待于进一步的研究。另外, 头状花序 大小的增幅小于头状花序数量的增幅, 这是否因头 状花序可发展空间有限而受到限制? 自然条件下, 黄帚革吾头状花序的昆虫寄生率很高, 至少可造成 $50 \%$ 左右的头状花序败育 (发育的种子被寄生昆虫 所食) , 故个体对头状花序数量的优先选择是否与此 相关? 黄帚䝴吾有性繁殖的这些特征对其通过根状 茎进行无性增殖将产生何种影响? 这些问题都有待 去研究, 这样才能对黄帚革吾有性繁殖有一个较为 深刻的认识。

\section{参 考 文 献}

Anne, C. W. , A.M. Baker, J.D. Thompson \& S.C.H. Barrett. 2000. Floral display in narcissus: variation in flower size and number at the species, population, and individual levels. International Journal of Plant Science, 161: 69 79 .

Ashman, T. L. \& M. S. Hitchens. 2000. Dissecting the causes of variation in intra-inflorescence allocation in a sexually polymorphic species, Fragaria virginiana (Rosaceae). American Journal of Botany, 87: $197 \sim 204$.

Ashman, T. L., J. Pacyna, C. Diefenderfer \& T. Leftwich. 2001. Size-dependent sex allocation in a gynodioecious wild strawberry: the effects of sex morph and inflorescence architecture. International Journal of Plant Sciences, 162: $327 \sim 334$.

Bayer, C. 1999. The bicolor unit - homology and transformation of an inflorescence structure unique to core Malvales. Plant Systematics and Evolution, 214: $187 \sim 198$.

Bawa, K. S. \& C. J. Webb. 1984. Flower, fruit and seed abortion in tropical forest trees: implications for the evolution of paternal and maternal reproductive patterns. American Journal of Botany, 71: $736 \sim 751$.

Bradley, D., O. Ratcliffe, C. Vincent, R. Carpenter \& E. Coen. 1997. Inflorescence commitment and architecture in Arabidopsis. Science, 275: $80 \sim 83$.

Brody, A. K. \& R. J. Mitchell. 1997. Effects of experimental manipulation of inflorescence size on pollination and pre-dispersal seed predation in the hummingbird-pollinated plant Ipomopsis aggregata. Oecologia, 110: 86 93.

Brunet, J. \& D. Charlesworth. 1995. Floral sex allocation in sequentially blooming plants. Evolution, 49: $70 \sim 79$.

Brunet, J. 1996. Male reproductive success and variation in fruit and seed set in Aquilegia caerulea (Ranunculaceae). Ecology, 77: $2458 \sim 2471$.

Cunningham, S. A. 1997. The effect of light environment, leaf area, and stored carbohydrates on inflorescence production by a rain forest understory palm. Oecologia, 111: $36 \sim 44$.

Diggle, P. K. 1995. Architectural effects and the interpretation of patterns of fruit and seed development. Annual Review of Ecology and Systematics, 26: $531 \sim 552$.

Diggle, P. K. 1997. Ontogenetic contingency and floral morphology: the effects of architecture and resource limitation. International Journal of Plant Sciences, 158 (Suppl. ) : 99 107.

Du, G. Z. (杜国祯), G. J. Sun (孙国均), X. Z. Wang (王
兮之) \& Z. H. Liu (刘振恒). 1999. The relationship between size-dependent reproduction and population density of Elymus nutans. Acta Prataculturae Sinica (草业学报), 8: $26 \sim 33$. (in Chinese with English abstract)

Du, G. Z. (杜国祯) \& G. Wang (王刚). 1998. Relationship between individual size and density of Elymus nutans population. Acta Phytoecologica Sinica (植物生态学报), 22: $552 \sim 558$. (in Chinese with English abstract)

Du, R. Q. (杜荣骞). 1999. Biostatistics. Beijing: China Higher Education Press. $117 \sim 127,177 \sim 193$. (in Chinese)

Emms, S. K., D. A. Stratton \& A. A. Snow. 1997. The effect of inflorescence size on male fitness: experimental tests in the andromonoecious lily, Zigadenus paniculatus. Evolution, 51: 1481 $\sim 1489$.

Fishbein, M. \& D. L. Venable. 1996. Evolution of inflorescence design: theory and data. Evolution, 50: $2165 \sim 2177$.

Germón, A. S., G. A. Krupnick \& A. G. Stephenson. 2001. Growth and resource allocation in Cucurbita pepo ssp. Texana: effects of fruit removal. International Journal of Plant Sciences, 162: $1089 \sim 1095$.

Guitión, J. 1994. Selective fruit abortion in Prunus mahaleb (Rosaceae) . American Journal of Botany, 81: $1555 \sim 1558$.

Harder, L. D. \& S. C. H. Barrett. 1995. Mating cost of large floral displays in hermaphrodite plants. Nature, 373: $512 \sim 515$.

Klinkhamer, P. G. L., E. Meelis, T. J. de Jong \& J. Weiner. 1992. On the analysis of size-dependent reproductive output in plants. Functional Ecology, 6: $308 \sim 316$.

Komatsu, M., M. Maekawa, K. Shimamoto \& J. Kyozuka. 2001. The LAX1 and FRIZZY PANICLE 2 genes determine the inflorescence architecture of rice by controlling rachis-branch and spikelet development. Developmental Biology, 231: $364 \sim 373$.

Laura, F.G., T. Cirigliano \& K. Gremski. 2002. The contribution of display size and dichogamy to potential geitonogamy in Campanula americana. International Journal of Plant Science, 163: $133 \sim 139$.

Lavee, S., L. Rallo, H. F. Rapoport \& A. Troncoso. 1999. The floral biology of the olive. II . The effect of inflorescence load and distribution per shoot on fruit set and load. Scientia Horticulturae, 82: $181 \sim 192$.

Lee, T. D. 1988. Patterns of fruit and seed production. In: Doust, L. L. ed. Plant reproductive ecology: patterns and strategies. Oxford: Oxford University Press. 179 202.

Liu, Z. J. (刘左军) , G. Z. Du (杜国祯) \& J. K. Chen (陈家 宽). 2002. Size-dependent reproductive allocation of Ligularia virgaurea in different habitats. Acta Phytoecologica Sinica (植物 生态学报), 26: 44 50. (in Chinese with English abstract)

Maes, T., M. van Montagu \& T. Gerats. 1999. The inflorescence architecture of Petunia hybrida is modified by the Arabidopsis thaliana Ap2 gene. Developmental Genetics, 25: 199 208.

Mazer, S. J. \& K. A. Dawson. 2001. Size-dependent sex allocation with flowers of the annual herb Clarkia unguiculata (Onagraceae) : ontogenetic and among-plant variation. American Journal of Botany, 88: 819 831.

Medrano, M., P. Guitián \& J. Guitián. 2000. Patterns of fruit and seed set within inflorescences of Pancratium maritimum (Amaryllidaceae): nonuniform pollination, resource limitation, or architectural effects? American Journal of Botany, 87: 493 501 .

Nakamura, R. R. 1986. Maternal investment and fruit abortion in Phaseolus vulgaris. American Journal of Botany, 73: 1049 1057. 
Ohara, M. \& S. Higashi. 1994. Effects of inflorescence size on visits from pollinators and seed set of Corydalis ambigua ( Papaveraceae). Oecologia, 98: $25 \sim 30$.

Proctor, J. T. A., D. C. Percival \& D. Louttit. 1999. Inflorescence removal affects root yield of American ginseng. Hortscience, 34: $82 \sim 84$

Schmid, B. \& J. Weiner. 1993. Plastic relationships between reproductive and vegetative mass in Solidago altissima. Evolution, 47: $61 \sim 74$.

Shan, B. Q. (单保庆), G. Z. Du (杜国祯) \& Z. H. Liu (刘 振恒). 2000. Clonal growth of Ligularia virgaurea: morphological responses to nutritional variation. Acta Phytoecologica Sinica (植物生态学报), 24: 46 51. (in Chinese with English abstract)

Singer, S., J. Sollinger, S. Maki, J. Fishbach, B. Short, C. Reinke, J. Fick, L. Cox, A. McCall \& H. Mullen. 1999. Inflorescence architecture: a developmental genetics approach. Botanical Review, 65: $385 \sim 410$.

Souer, E., A. van der Krol, D. Kloos, C. Spelt, M. Bliek, J. Mol \& R. Koes. 1998. Genetic control of branching pattern and floral identity during Petunia inflorescence development. Development, 125: $733 \sim 742$.

Thomson, J. D. 1985. Pollination and seed set in Diervilla lonicera (Caprifoliaceae) : temporal patterns of flower and ovule development. American Journal of Botany, 72: $737 \sim 740$.
Tucker, S. C. \& J. Grimes. 1999. The inflorescence: introduction. Botanical Review, 65: $303 \sim 316$.

Warringa, J.W., R. de Visser \& A.D. H. Kreuzer. 1998. Seed weight in Lolium perenne as affected by interactions among seeds within the inflorescence. Annals of Botany, 82: 835 841 .

Weiner, J. 1988. The influence of competition on plant reproduction. In: Doust, L. L. ed. Plant reproductive ecology: patterns and strategies. Oxford: Oxford University Press. $228 \sim 245$.

Willson, M. F. \& P.W. Price. 1977. Evolution of inflorescence size in Asclepias (Asclepiadaceae). Evolution, 31: $495 \sim 511$.

Wolfe, L. M. 1992. Why does the size of reproductive structures decline through time in Hydrophyllum appendiculatum ( $\mathrm{Hy}-$ drophyllaceae)? Developmental constraints vs. resource limitation. American Journal of Botany, 79: 1286 1290 .

Worley, A. C., A. M. Baker, J. D. Thompson \& S. C. H. Barrett. 2000. Floral display in Narcissus: variation in flower size and number at the species, population, and individual levels. International Journal of Plant Sciences, 161: 69 79 .

Wu, Z. Y. (吴征镒). 1980. Vegetation of China. Beijing: Science Press. 624 649. (in Chinese)

Zhang, D. Y. (张大勇) \& X. H. Jiang (姜新华). 2001. Mating system evolution, resource allocation, and genetic diversity in plant. Acta Phytoecologica Sinica (植物生态学报), 25: 130 143. (in Chinese with English abstract) 